\title{
Percepciones de Estudiantes y Docentes: Evaluación Formativa en Proyectos de Aprendizaje Tutorados
}

\section{Perceptions of Students and Teachers: Formative Evaluation in Tutored Learning Projects}

\author{
Lurdes Martínez-Mínguez ${ }^{1 *}$ \\ Laura Moya Prados ${ }^{1}$ \\ Carolina Nieva Boza ${ }^{1}$ \\ Dolors Cañabate Ortiz ${ }^{2}$ \\ ${ }^{1}$ Universitat Autònoma de Barcelona, España \\ ${ }^{2}$ Universitat de Girona, España
}

\begin{abstract}
Este artículo presenta los resultados de una investigación educativa en conexión entre el mundo académico y profesional en el grado de maestro/a de dos universidades catalanas (UAB, $\mathrm{UdG}$ ). El objetivo de esta investigación es analizar las percepciones de profesorado, maestros-psicomotricistas y estudiantes sobre la evaluación formativa en Proyectos de Aprendizaje Tutorados (PAT) para facilitar la adquisición de competencias profesionales psicomotrices en la formación inicial de maestros. Se ha desarrollado en 2 asignaturas obligatorias de 2 titulaciones, con una muestra de 170 estudiantes, 2 profesoras universitarias y 7 maestrospsicomotricistas de escuelas. En ambas asignaturas se ha desarrollado una actividad de aprendizaje y evaluativa: documentación sesión psicomotricidad en grupo, en formato PAT. El estudio se enmarca dentro del paradigma interpretativo, también denominado cualitativo, naturalista o humanista. Se ha utilizado dos instrumentos de investigación: un cuestionario de escala tipo Likert y dos grupos de discusión. Se ha procedido al análisis estadístico a través de media aritmética y la desviación estándar Para el análisis cualitativo de los grupos de discusión se ha utilizado el análisis categorial. Principalmente los resultados y conclusiones muestran que: a) los estudiantes están satisfechos de forma global con la evaluación y coevaluación realizada en el PAT; b) esta evaluación ha favorecido la adquisición de competencias profesionales; c) la retroalimentación permite a los estudiantes ser más conscientes de su proceso y de sus errores; y d) estudiantes, profesoras y maestros coinciden en la necesidad de revisar los instrumentos de evaluación del PAT y evaluarlos a través de rúbricas.
\end{abstract}

Palabras clave: Enseñanza superior; Innovación educativa; Evaluación formativa; Formación de docentes; Tutoría.

This article presents the results of some educational research about connecting the academic and professional environments in the education degree within two Catalan universities (UAB, UdG). The goal of this study is to analyse perceptions of faculty members, psychomotricity teachers and students about formative assessment in Project of Oriented Learning (POL) to facilitate the acquisition of psychomotor professional skills in pre-service education. This research has been developed in two obligatory subjects in two Spanish university degrees, with a sample of 170 students, 2 lecturers and 7 psychomotricity teachers. Both subjects have developed an assessment and learning activity called: documentation psychomotor session in groups, in POL format. This study is conceived within the interpretative approach, also called qualitative, naturalist or humanist. Two research techniques are used; a Likert scale questionnaire and two focus groups. Statistical analysis was carried out through arithmetic mean and the standard deviation. The qualitative analysis of the focus groups has been done using the categorical analysis. The resulting data and

*Contacto: lurdes.martinez@uab.cat

issn: 1989-0397

www.rinace.net/riee/

https://revistas.uam.es/riee
Recibido: $\quad 14$ de enero de 2019

$1^{\text {a }}$ Evaluación: 03 de marzo de 2019

$2^{\text {a }}$ Evaluación: 01 de abril de 2019

Aceptado: $\quad 08$ de abril de 2019 
the conclusions reveal that students: a) are generally satisfied with the assessment and shared or co-assessment developed in the POL; b) this assessment has benefited their acquisition of professional skills; c) feedback allows students to be more aware of their process and their mistakes; and d) students, lecturers and teachers agree on the need to review the POL assessment instruments and assess them through rubrics.

Keywords: Higher education; Educational innovations; Formative assessment; Teacher education; Tutoring.

\section{Introducción}

Cada vez hay más problemas derivados del sedentarismo en la juventud de nuestra sociedad. En las ciudades los niños salen menos a jugar al parque o a la calle y, a su vez, las pantallas están ocupando muchas horas del día de éstos. Cuando los niños de hoy en día tengan alrededor de 70 años habrán pasado un promedio de entre siete y diez años frente a una pantalla, siendo esta la segunda actividad más realizada después del dormir (Trenchi, 2014).

Por otro lado, es sabido que el movimiento y el juego libre son la mejor forma de aprendizaje y desarrollo de los niños de cero a seis años (Bueno, 2017; Sassano, 2010). Por lo que las escuelas juegan un papel importante para acoger, respetar y acompañar la educación por el movimiento en todas las situaciones educativas y en los diferentes espacios (Cortés y Moya, 2018; Martínez-Mínguez et al., 2017).

En el Estado Español los currículos de Educación Infantil están organizados por áreas y una de ellas suele estar relacionada con el conocimiento de uno mismo a través del cuerpo y el movimiento, tanto en actividades de vida cotidiana como en momentos específicos relacionados con la educación psicomotriz (Martínez-Mínguez, Rota y Anton, 2017). En el primer ciclo de educación primaria también se muestra desde el área de Educación Física la importancia de este movimiento y conocimiento corporal de una forma lúdica y autónoma (Cañabate, Colomer y Oliveras, 2018). Atender a esta área hace necesaria una formación de maestros capacitados para impartirla en su quehacer profesional tanto a nivel teórico como práctico.

La experiencia docente de algunos autores de este artículo remite a que parece que no siempre los maestros de educación infantil y especialistas de educación física, después de su formación inicial, se sienten suficientemente preparados para realizar la intervención psicomotriz. Esta problemática adopta diferentes soluciones en los centros educativos. A veces se pide al maestro especialista de educación física que imparta la psicomotricidad en los cursos de educación infantil, cuando su formación inicial hace referencia a la etapa de 6 a 12 años. Otras veces es impartida por algún maestro de educación infantil que no realice tutoría. Otra posibilidad es que la realice algún maestro con formación específica a través de un postgrado o máster relacionado con la psicomotricidad. No obstante, se considera que sería conveniente que el maestro de educación infantil fuera el responsable de la docencia de las tres áreas del currículum de esta etapa expuestas en la Orden ECI/3960/2007, de 19 de diciembre (Conocimiento de uno mismo y autonomía personal, Conocimiento del entorno, y Lenguajes: comunicación y representación). 


\section{Revisión de la literatura}

El informe de Eurydice (2002) sobre la profesión docente en Europa señalaba cómo conseguir que el profesorado se capacite y realice una docencia de forma efectiva. Esto exige aumentar la calidad de la formación inicial de los docentes, con unas mejores competencias que respondan a las demandas sociales presentes y futuras. La reorganización del currículo español de Educación Superior de los últimos quince años se ha visto afectado entre otros, por: La Declaración de Bolonia (1999); los acuerdos del Comunicado de Berlín (2003); y por el Documento-Marco "La integración del Sistema Universitario Español en el EEES" (MEC, 2003). El contenido de estos documentos está todavía determinando que, en los planes de estudio relacionados con educación, no sólo que se modificara el currículo formativo basado en competencias generales y específicas, sino también el papel del profesor universitario como desarrollador de competencias que ayuden a ser un buen docente al estudiante (OCDE, 2002; Perrenoud, 2004).

Según Martínez-Mínguez y Flores (2014), el alumnado y profesorado de Enseñanza Superior opinan que la conexión entre el mundo académico y el mundo profesional, junto con la adquisición de competencias de las actuales titulaciones universitarias, no siempre son suficientemente satisfactorias. En su estudio se apunta que se prima que el estudiantado durante su formación inicial desarrolla más las capacidades cognitivas como memorizar y recordar, que las de aplicar o analizar en entornos profesionales auténticos. En consecuencia, en la formación inicial de maestros es necesaria la utilización de metodologías docentes que favorezcan la creación de puentes o conexiones entre teoríapráctica. Se necesitan asignaturas con una metodología activa, participativa y significativa, tal como expone Cano (2012, p.14-15): "los diseños por competencias obligan a buscar fórmulas para que los estudiantes integren y apliquen saberes. Eso significa que aumenta el sentido que poseen los casos, los proyectos, las simulaciones, las prácticas, etc."

Los PAT son una modalidad de proyectos que conectan bien la relación teoría-práctica. Algunos autores consideran que los PAT son una "buena práctica" (López y Vicente, 2015; Martínez-Mínguez, 2015). Meyer (2002) define un PAT como una forma de trabajo en la que grupos de estudiantes son dirigidos para solucionar problemas que se pueden plantear en su desempeño profesional. Se basan en el aprendizaje cooperativo para favorecer el desarrollo de competencias genéricas y profesionales, el liderazgo, la autoeficacia, la capacidad crítica y de comunicación (Barba, Martínez y Torrego, 2012) y valores como la justicia, la solidaridad, la responsabilidad y la cooperación (Guilarte, Marbán y Miranda, 2008). Sería interesante comprobar si los PAT pueden contribuir a mejorar la formación inicial de competencias profesionales psicomotrices de maestros.

Para Álvarez, García, Gil y Romero (2004) en los PAT se concretan tres características: Ser una propuesta que permita al estudiantado aprender de forma autónoma y en diferentes escenarios, bajo la supervisión del profesorado; su principal aprendizaje se basa en el "cómo hacer", desarrollando así habilidades y competencias profesionales; y el alumnado es el responsable de su propio aprendizaje, si bien en este proceso tienen la colaboración del profesor o profesora.

Martínez-Mínguez y Moya (2017) consideran que tiene un buen resultado incorporar PAT a programas de asignaturas que buscan interacciones entre contenidos bien seleccionados y organizados con la realización de experiencias y actividades que permitan 
entenderlos en profundidad, cultivando operaciones cognitivas superiores y construyendo sentido sobre lo que se aprende desarrollándolo en contextos de práctica real. Por lo que podría ser interesante crear equipos docentes incorporando a maestros de centros educativos en ejercicio a colaborar de alguna manera en la docencia de asignaturas de Enseñanza Superior.

En relación a las competencias, no solamente hay que pensar cómo desarrollarlas sino también cómo evaluarlas. Otro de los aspectos clave del cambio que lleva implícito trabajar por competencias en la Enseñanza Superior es la forma de entender los sistemas de evaluación del alumnado. La evaluación condiciona los procesos de aprendizaje (Zabalza, 2002). Algunos autores avalan que metodologías activas y sistemas de evaluación formativa inciden positivamente en el desarrollo de mayores niveles de aprendizaje en el alumnado (Biggs, 1999; Brockbank y McGill, 2002; Brown y Glasner, 2000; Colomer, Serra, Cañabate y Serra, 2018; San Martín, 2012), y concretamente en aprendizajes auténticos y significativos (Martínez-Mínguez, 2016).

Por evaluación formativa puede entenderse todo proceso de evaluación que su finalidad principal sea mejorar los procesos de enseñanza-aprendizaje que se desarrollan (LópezPastor, 2009). Numerosos trabajos confirman la importancia de lograr un carácter formativo en la evaluación (Galván y Farías, 2018; Valvanuz y Salcines, 2018) frente a una evaluación sumativa y finalista (Álvarez, 2003; Biggs, 1999; Bonson y Benito, 2005; Brown y Glasner, 2000; López et al. 2009; Salinas, 2002; Zabalza, 2002).

Cada vez se hace más necesario conocer y aplicar un sistema de evaluación continuo, variado y formativo (Gómez y Quesada, 2017; Inda, Álvarez y Álvarez, 2008; Hortigüela, Pérez-Pueyo y López-Pastor, 2015), dentro de lo que se ha venido a denominar la aparición de una nueva cultura de la evaluación (Rust, 2007).

Mas (2011), en su estudio sobre el perfil competencial del profesor universitario, concreta seis competencias relacionadas con su función docente y algunas de las unidades competenciales relacionadas con la evaluación son: (1) diseñar el plan de evaluación del aprendizaje y los instrumentos necesarios; (2) aplicar el dispositivo de evaluación de acuerdo al plan evaluativo establecido; (3) verificar el logro de aprendizajes de los alumnos; (4) evaluar los componentes del proceso de enseñanza-aprendizaje; (5) promover y utilizar técnicas e instrumentos de autoevaluación discente; (6) tomar decisiones basándose en la información obtenida; (7) implicarse en los procesos de coevaluación; y (8) afrontar los deberes y dilemas éticos de la evaluación.

Según Angulo (2008), el reto está en diseñar actividades de evaluación pertinentes y contextualizadas que contribuyan a desarrollar competencias clave para educar crítica y reflexivamente a los profesionales del futuro, capacitándolos como generadores de su propio conocimiento para comprender y transformar la realidad.

La evaluación debe convertirse en una estrategia para mejorar y favorecer los aprendizajes durante todo el proceso de formación (Romero, Fraile, López y Castejón, 2014). Para García-Sanz (2014), la evaluación se caracteriza por activar al estudiante hacia el aprendizaje, orientándolo hacia su mejora e informándole de forma continua y no únicamente al final, sobre sus progresos en relación a sus competencias. Se trata de pasar de una "evaluación del aprendizaje" a una "evaluación para el aprendizaje" (López-Pastor, 
2009), e incluso a una evaluación "como aprendizaje" (Torrance, 2007), ya que una buena evaluación puede ser la mejor actividad de aprendizaje.

Ibarra, Rodríguez y Gómez (2012), definen la evaluación orientada al aprendizaje:

Como un constructo teórico, basado en la práctica universitaria, que hace hincapié en considerar la evaluación como un proceso interrelacionado con el aprendizaje, a partir del cual, no sólo se puede extraer información útil para el estudiante, sino que se posibilita la promoción del desarrollo de competencias valiosas para su presente académico y desarrollo profesional. (p. 210)

Para Álvarez (2008), la evaluación orientada al aprendizaje debe apoyarse en tres aspectos fundamentales: plantear las actividades de evaluación como tareas de aprendizaje; implicar a los estudiantes en la evaluación; ofrecer los resultados de la evaluación a modo de feedback. Es decir, que la evaluación debe pasar a formar parte del propio proceso de formación con un feedback continuo que informe al estudiante en qué punto de su proceso de aprendizaje y adquisición de competencias se encuentra (Cano, 2012; Escudero, 2010; Ferguson, 2011; Sadler, 2010).

La evaluación de competencias requiere el empleo de distintos instrumentos de recogida de información complementarios sobre el progreso del alumnado, que nos informen de forma completa y permitan emitir juicios de valor acertados, precisos y justos (LópezRuiz, 2011). Según García-Sanz (2014) es necesario que sean instrumentos acordes con las competencias a mesurar y que atiendan al principio de multivariedad y triangulación instrumental. Además, deben reflejar aquellos contenidos sujetos a evaluación que son verdaderamente importantes para el aprendizaje del alumnado, poniendo de manifiesto la adquisición de competencias. A su vez estos instrumentos han de constituir una herramienta de mejora para el propio aprendizaje y de apoyo al proceso de enseñanza.

Es aconsejable otorgar una mayor participación a todos los agentes implicados en el proceso evaluativo utilizando procedimientos de autoevaluación y coevaluación entre profesorado y alumnado o recíprocamente entre alumnado (Bretones, 2008; Echeberría, 2002; Falchikov, 2005; Lin y Lai, 2013; Medina-Rivilla, Domínguez-Garrido y SánchezRomero, 2013; Rodríguez, Ibarra y Gómez, 2011; Taras, 2002 y 2010; Trevitt, Breman y Stocks, 2012).

Una vez introducido el tema de investigación y revisada la literatura, se concreta que el objetivo a desarrollar en este artículo es: Analizar las percepciones de profesorado, maestros-psicomotricistas y estudiantes sobre la evaluación formativa en PAT para facilitar la adquisición de competencias profesionales psicomotrices en la formación inicial de maestros.

\section{Método}

El enfoque metodológico de este estudio es mixto combinando metodologías de investigación cuantitativa y cualitativa.

La finalidad de este estudio es interpretar y comprender los fenómenos educativos más que aportar una explicación de tipo causal. Por lo que esta investigación se encuentra enmarcada dentro del paradigma interpretativo, el cual también se denomina cualitativo, naturalista o humanista (Bisquerra, 2014; Albert, 2007). 
Según Hernández, Fernández y Baptista (2010), el enfoque metodológico de este estudio es mixto, de diseño incrustado concurrente de modelo dominante (DIAC). En él se reconoce que un método (cuantitativo o cualitativo) posee menor prioridad y está incrustado o anidado dentro del que se considera central. En esta investigación la predominancia es cualitativa y el método cuantitativo se incrusta en ella. Coincidiendo, con Creswell (2009) en que un estudio básicamente cualitativo puede enriquecerse con datos cuantitativos descriptivos de la muestra. Este método proporciona una visión más amplia del fenómeno estudiado que si se usara un solo método.

Los datos recolectados por ambos métodos se han comparado en la fase de análisis.

\subsection{Contexto}

La investigación se ha desarrollado en dos asignaturas de dos titulaciones de dos universidades. Por un lado, la asignatura de Educación Psicomotriz en los centros de Educación Infantil de cuarto curso del Grado en Educación Infantil de la "Universitat Autònoma de Barcelona” (UAB). Por otro lado, la asignatura de Educación Física I de segundo curso del Grado de Maestro en Educación Primaria de la "Universitat de Girona" (UdG). Ambas asignaturas son obligatorias en el plan de estudios de la titulación correspondiente.

Cuadro 1. Descripción de los instrumentos de evaluación formativa utilizados en el PAT

\begin{tabular}{|c|c|c|c|c|}
\hline \multirow{2}{*}{ INSTRUMENTO } & \multirow{2}{*}{ DESCRIPCIÓN } & \multicolumn{3}{|c|}{ EVALUADOR } \\
\hline & & $\mathrm{P}$ & $\mathrm{M}$ & $\mathrm{E}$ \\
\hline $\begin{array}{c}\text { Pauta sesión en la } \\
\text { universidad }\end{array}$ & $\begin{array}{l}\text { Pauta que permite evaluar de manera individual al } \\
\text { estudiante durante la sesión de psicomotricidad que } \\
\text { realiza con sus compañeros en la universidad. }\end{array}$ & $\mathrm{X}$ & & \\
\hline $\begin{array}{c}\text { Pauta sesión en la } \\
\text { escuela }\end{array}$ & $\begin{array}{l}\text { Pauta que permite evaluar a los estudiantes de } \\
\text { manera grupal durante la sesión de psicomotricidad } \\
\text { que realizan con los niños en la escuela. }\end{array}$ & & $\mathrm{X}$ & \\
\hline $\begin{array}{l}\text { Rúbrica exposición } \\
\text { PAT y } \\
\text { documentación } \\
\end{array}$ & $\begin{array}{l}\text { Rúbrica que permite evaluar de manera grupal } \\
\text { durante la exposición del PAT y su documentación } \\
\text { pedagógica. }\end{array}$ & $\mathrm{X}$ & $\mathrm{X}$ & \\
\hline $\begin{array}{l}\text { Escala } \\
\text { Autoevaluación } \\
\text { Competencias }\end{array}$ & $\begin{array}{l}\text { Escala que permite al estudiante autoevaluar las } \\
\text { competencias profesionales psicomotrices que ha } \\
\text { trabajado durante el PAT. Ésta es contestada al } \\
\text { inicio de la asignatura, después de realizar la sesión } \\
\text { en la escuela y al final. }\end{array}$ & & & $\mathrm{X}$ \\
\hline $\begin{array}{l}\text { Hipótesis sesión } \\
\text { universidad }\end{array}$ & $\begin{array}{l}\text { Redacción de posibles sucesos que pueden } \\
\text { transcurrir en la sesión de la universidad. }\end{array}$ & & & $\mathrm{X}$ \\
\hline $\begin{array}{l}\text { Hipótesis sesión } \\
\text { escuela }\end{array}$ & $\begin{array}{l}\text { Redacción de posibles sucesos que pueden } \\
\text { transcurrir en la sesión de la escuela. }\end{array}$ & & & $\mathrm{X}$ \\
\hline
\end{tabular}

Nota: $\mathrm{P}=$ Profesor universitario; $\mathrm{M}=$ Maestro-Psicomotricista; $\mathrm{E}=$ Estudiantes.

Fuente: Elaboración propia.

En estas asignaturas se ha desarrollado una actividad de aprendizaje y evaluación llamada: "Documentación sesión psicomotricidad en grupo". Esta es una actividad basada en PAT que concluye con una exposición en formato documentación pedagógica. Los estudiantes, después de haber recibido el marco teórico sobre qué significa, fundamenta y conforma la educación psicomotriz, en grupo diseñan y llevan a la práctica una sesión de psicomotricidad. Primero la realizan con sus compañeros de la universidad y, después, en un contexto real con un grupo clase de una escuela. Tanto para la elaboración de estas sesiones como para su desarrollo están tutorizados por la profesora de la universidad y 
acompañados por el psicomotricista o el especialista de educación física de la escuela en la que van a intervenir. En la sesión práctica en la universidad y en la presentación final de la documentación reciben evaluación formativa a través de feedback, no sólo del docente, sino también de sus compañeros. Además, en la sesión práctica con los niños reciben feedback por parte del maestro-psicomotricista de la escuela. Para poder llevar a cabo esta evaluación formativa en el PAT, se utilizan diversos instrumentos usados por los diferentes participantes (cuadro 1).

\subsection{Muestra}

La muestra investigada pertenece al tipo no probabilístico (Hernández et al. 2010) y por conveniencia (Battaglia, 2008a). Los participantes representan un subgrupo de la población en la que la elección de éstos responde a características de la investigación según los casos disponibles a los que se tiene acceso.

Los sujetos o participantes que componen la muestra de este estudio han sido de 3 tipos diferentes: estudiantes, profesorado universitario y maestros-psicomotricistas de escuelas (Cuadro 2).

Cuadro 2. Muestra del estudio

\begin{tabular}{lccccc}
\hline PARTICIPANTES & UNIVERSIDAD & $\begin{array}{c}\text { MUESTRA } \\
\text { TOTAL SEGÚN } \\
\text { UNIVERSIDAD } \\
\text { Y TIPO DE } \\
\text { PARTICIPANTE }\end{array}$ & $\begin{array}{c}\mathbf{N}^{\mathbf{2}} \\
\text { HoMBRES }\end{array}$ & $\begin{array}{c}\text { No } \\
\text { MUJERES }\end{array}$ & $\begin{array}{c}\text { MUESTRA } \\
\text { TOTAL SEGÚN } \\
\text { EL TIPO DE } \\
\text { PARTICIPANTE }\end{array}$ \\
\hline Estudiantes & $\mathrm{UAB}$ & 109 & 1 & 108 & \multirow{2}{*}{170} \\
\hline Profesores & $\mathrm{UdG}$ & 61 & 51 & 10 & 2 \\
universitarios & $\mathrm{UAB}$ & 1 & 0 & 1 & \multirow{2}{*}{7} \\
\hline Maestros- & $\mathrm{UdG}$ & 1 & 0 & 1 & 2 \\
psicomotricistas & $\mathrm{UAB}$ & 4 & 1 & 3 & 2 \\
\hline
\end{tabular}

Fuente: Elaboración propia.

En relación a los estudiantes, de una población de 220 estudiantes matriculados en las asignaturas, finalmente la muestra de estudio la han compuesto 170 de éstos. El número inferior de participantes de la muestra respecto a la población se ha producido por el carácter voluntario de la participación, por no completar todas las respuestas del cuestionario o por no cumplir con el calendario fijado.

Por lo que se refiere a los docentes, como profesorado universitario se conformó una muestra de dos profesores (uno para los dos grupos de docencia de la UAB y otro para el único grupo de docencia de la UdG). Como maestros-psicomotricistas de escuelas participaron siete maestros-psicomotricistas de centros de Educación Infantil y Primaria (cuatro para la UAB y tres para la UdG).

\subsection{Instrumentos de obtención de información}

El PAT se ha valorado como actividad de aprendizaje y evaluación a través de dos instrumentos: Cuestionario y grupo de discusión.

El primer instrumento utilizado en esta investigación es el Cuestionario sobre el PAT como experiencia de "Buena Práctica" (CPATEBP) basado en el Cuestionario sobre 
metodología y evaluación en Formación Inicial en Educación Física, validado por Castejón, Santos y Palacios (2015).

El CPATEBP consta de 16 preguntas y para este estudio se han analizado dos de ellas (Cuadro 3). A éstas el estudiante debía responder a una escala de tipo Likert dónde se identificaron cinco puntos de una escala ordinal, con valores numérico. El valor O se corresponde a la opción nada, el 1 a poco, el 2 a algo, el 3 a bastante y el 4 a mucho, además de la posibilidad No sabe / No contesta. Todas las preguntas debían responderse por los estudiantes al finalizar el PAT que coincidía con el final de la asignatura.

Cuadro 3. Preguntas del CPATEBP relacionadas con la evaluación formativa del PAT PREgUNTAS ANALIZADAS EN ESTE ESTUDIO

3.- ¿La evaluación que se ha planteado favorece la adquisición de competencias profesionales? 9.- Señala la satisfacción global en relación a la evaluación del PAT

Fuente: Elaboración propia.

Respecto al segundo instrumento, se han realizado dos grupos de discusión entendidos como una modalidad de entrevista grupal (Del Rincón, Arnal, Latorre y Sans, 1995; Hernández et al., 2010). Se ha dinamizado por un coordinador quién a través de un protocolo con diferentes niveles de estructuración, provoca la discusión y la dirige hacia la información deseada. Se trata de potenciar la participación de los asistentes y evidenciar los argumentos enfrentados en el debate. Los participantes han sido:

- Primer grupo de discusión: Estudiantes de las dos universidades de la UAB (seis participantes de la $\mathrm{UAB}$ y seis de la $\mathrm{UdG}$ ).

- Segundo grupo de discusión: Profesorado universitario (uno de la UAB y otro de la $\mathrm{UdG}$ ) y maestros-psicomotricistas (cuatro de la UAB y 3 de la $\mathrm{UdG}$ ).

Cuadro 4. Criterios de heterogeneidad de la muestra del primer grupo de discusión

\begin{tabular}{lc}
\hline ATRIBUTO & Valor \\
\hline UNIVERSIDAD & UAB \\
UdG \\
\hline EDAD & Hasta 19 años (UdG) y 21 (UAB) \\
ESTUDIOS PREVIOS & Co o más (UdG) y 22 o más (UAB) \\
\hline \multirow{2}{*}{ EXPERIENCIA DOCENTE } & Bachillerato \\
\hline NOTA MEDIA DEL EXPEDIENTE & Sí \\
ACADÉMICO & No \\
\hline & Suspendido / Aprobado / Bien / Notable bajo \\
& Notable alto / Excelente \\
MAESTRO-PSICOMOTRICISTA QUE HA & Maestro-Psicomotricista 1 \\
ACOMPAÑADO LA SESIÓN DEL PAT & Maestro-Psicomotricista 2 \\
& Maestro-Psicomotricista 3 \\
& Maestro-Psicomotricista 4 \\
& Maestro-Psicomotricista 5 \\
SEXO & Maestro-Psicomotricista 6 \\
& Maestro-Psicomotricista 7 \\
\hline
\end{tabular}

Fuente: Elaboración propia. 
La selección de la muestra del primer grupo fue a través de voluntarios. A esta clase de muestra también se le puede llamar autoseleccionada, ya que las personas responden a una invitación (Battaglia, 2008b). De 21 voluntarios se seleccionaron 12 a partir de criterios de homogeneidad (matriculados en las asignaturas de este estudio y heterogeneidad (universidad, edad, estudios previos, experiencia docente, nota media del expediente académico, maestro-psicomotricista que ha acompañado la sesión del PAT y sexo).

La selección de la muestra del segundo grupo es de tipo homogénea ya que el propósito de éste se centra en el estudio de una temática concreta (Hernández et al. 2010, Miles y Huberman, 1994), es por ello por lo que todos sus componentes participan como docentes en el PAT. Como características de los participantes de este grupo de discusión se destaca la universidad de procedencia, tipo de docencia, titulación y sexo:

Cuadro 5. Características de la muestra del segundo grupo de discusión

\begin{tabular}{ll}
\hline \multicolumn{1}{c}{ ATRIBUTO } & \multicolumn{1}{c}{ VALOR } \\
\hline \multirow{2}{*}{ UNIVERSIDAD } & UAB \\
& UdG \\
\hline \multirow{2}{*}{ TIPO DE DOCENCIA } & Profesor universidad \\
& Maestro-psicomotricista escuela \\
\hline \multirow{2}{*}{ TITULACIÓN } & Grado en Educación Infantil \\
\hline \multirow{2}{*}{ SEXO } & Grado en Educación Primaria \\
\hline
\end{tabular}

Fuente: Elaboración propia.

En referencia a la estructura de los dos grupos de discusión, éstos estaban compuestos cada uno de ellos por cinco puntos:

- Metodología: Se preguntaba sobre aspectos organizativos del PAT como puede ser el trabajo en equipo, la distribución de las sesiones, etc.

- Competencias: Se hace referencia a la adquisición de las competencias profesionales psicomotrices a partir del PAT. También sobre el acompañamiento de los docentes.

- Evaluación: Se ha tratado aspectos relacionados con la evaluación formativa como el tiempo destinado a la evaluación, el feedback, la valoración de los instrumentos, etc.

- Prospectiva: Se caracteriza por indagar la transferencia del PAT a otras asignaturas o al mundo laboral de los futuros maestros.

- Conclusiones: Se analizan los aspectos claves y débiles del PAT. También sobre sus aspectos a mejorar.

\subsection{Análisis de datos}

El análisis cuantitativo de las dos preguntas del CPATEBP se ha realizado por dos procedimientos de carácter estadístico: A través de puntuaciones medias como es el promedio aritmético de una distribución (Hernández et al., 2010) y la desviación estándar como el promedio de desviación de las puntuaciones con respecto a la media (Jarman, 2013). También se ha realizado una comparación de medias entre los subgrupos de estudiantes a través de una prueba T-Test para ver si existe alguna diferencia 
estadísticamente significativa. Los datos se han analizado con el programa Excel-2010 y el software de análisis cuantitativo IBM Statistical Package for de Social Sciences (SPSS) versión 20.

Para el análisis cualitativo de los grupos de discusión se ha utilizado el análisis categorial (Rodríguez, Gil y García, 1999) Los datos generados se registraron como notas de campo, grabación de audio y grabación de video. El texto de la transcripción se ha analizado a través del programa cualitativo Nudist N-Vivo $11 \circledR$. Su análisis se ha realizado siguiendo una lógica mixta que combina un proceso de categorización deductivo-inductivo (Gibbs, 2012).

\section{Resultados}

A continuación, se muestran los resultados obtenidos en la pregunta 3 y 9 del CPATEBP que los estudiantes han contestado.

Cuadro 6. Puntuaciones medias y desviación estándar preguntas 3 y 9 CPATEBP

\begin{tabular}{|c|c|c|c|c|c|c|c|}
\hline \multirow{2}{*}{ Pregunta CPATEBP } & \multicolumn{2}{|c|}{ UAB } & \multicolumn{2}{|c|}{ UDG } & \multicolumn{2}{|c|}{ TOTAL } & \multirow{2}{*}{$\begin{array}{l}\text { SIGN }(2- \\
\text { COLAS })\end{array}$} \\
\hline & $\mathrm{M}$ & DT & $\mathrm{M}$ & DT & $\mathrm{M}$ & DT & \\
\hline $\begin{array}{l}\text { 3.- ¿La evaluación que se ha } \\
\text { planteado favorece la adquisición } \\
\text { de competencias profesionales? }\end{array}$ & 3,52 &, 70 & 3,23 &, 72 & $3,4+1$ &, 72 & $\begin{array}{c}, 011 \\
(\mathrm{p}<0,05)\end{array}$ \\
\hline $\begin{array}{l}\text { 9.- Señala la satisfacción global en } \\
\text { relación a la evaluación del PAT. }\end{array}$ & 3,16 &, 80 & 3,12 & ,68 & 3,14 &, 75 & $\begin{array}{c}, 517 \\
(p>0,05)\end{array}$ \\
\hline
\end{tabular}

Fuente: Elaboración propia.

Los estudiantes de ambas universidades están bastante satisfechos de forma global con la evaluación realizada en el PAT (3,14 sobre 4). También, consideran que la evaluación realizada favorece bastante la adquisición de competencias profesionales (3,41 sobre 4).

Si analizamos de forma separada a los estudiantes de ambas universidades, se destacan dos aspectos. Por una parte, respecto a la satisfacción global en relación a la evaluación del PAT no existen diferencias significativas en las puntuaciones de los estudiantes de ambas universidades $(\mathrm{UAB}=3,16$ y $\mathrm{UdG}=3,12)$. Por otra parte, se puede apreciar una diferencia respecto a la percepción de la evaluación del PAT como favorecedora de adquisición de competencias. En este caso, los estudiantes de la UAB se muestran ligeramente más satisfechos que los de la $\mathrm{UdG}(\mathrm{M}=3,52$ y $\mathrm{M}=3,23$, respectivamente) siendo esta diferencia $($ dif $=, 29)$ estadísticamente significativa $(\mathrm{p}<0,05)$.

A continuación, se muestran los resultados cualitativos obtenidos de los grupos de discusión que amplían, profundizan y matizan la información cuantitativa acabada de exponer por parte de los estudiantes. Primeramente, se expone el sistema de categorización y el número de referencias de cada categoría. Posteriormente, se aportan citas textuales de la transcripción por lo que pueden tener alguna incongruencia gramatical, que se identifican con números para reservar el anonimato de los participantes.

En referencia al sistema de categorización, se han concretado una dimensión (Evaluación formativa en el PAT para facilitar la adquisición de competencias profesionales) y cinco categorías (Cuadro 7), cada una de ellas correspondiente a los tres tipos participantes e informantes (profesor universidad, maestro-psicomoticista y estudiante). 
Cuadro 7. Descripción de las categorías del estudio

\begin{tabular}{ll}
\hline \multicolumn{1}{c}{ CATEGORÍA } & \multicolumn{1}{c}{ DESCRIPCIÓN } \\
\hline $\begin{array}{l}\text { Aportación de la evaluación formativa } \\
\text { al proceso de aprendizaje. }\end{array}$ & $\begin{array}{l}\text { Aspectos en los que la evaluación formativa contribuye } \\
\text { al aprendizaje y competencias del estudiantado. }\end{array}$ \\
\hline $\begin{array}{l}\text { Coherencia entre la tutorización y la } \\
\text { evaluación formativa }\end{array}$ & $\begin{array}{l}\text { Coherencia entre el proceso de tutorización del } \\
\text { estudiante y su evaluación. }\end{array}$ \\
\hline Instrumentos de evaluación utilizados & $\begin{array}{l}\text { Aspectos relevantes de los instrumentos de evaluación } \\
\text { utilizados en el PAT. }\end{array}$ \\
\hline $\begin{array}{l}\text { Participación del estudiante } \\
\text { Tiempo destinado a la evaluación }\end{array}$ & $\begin{array}{l}\text { Papel del estudiantado en el proceso de evaluación. } \\
\text { Tiempo destinado a realizar la evaluación del PAT. }\end{array}$ \\
\hline
\end{tabular}

Fuente: Elaboración propia.

En la figura 1 se muestra el número de referencias (referen.) obtenidas para cada categoría e indicador para poder mostrar los resultados desde una perspectiva global.

\begin{tabular}{|c|c|c|c|}
\hline \multicolumn{4}{|l|}{ Nodos } \\
\hline Nombre & बल & Recursos & Referen \\
\hline E. Evaluación formativa en Proyectos de Aprendizaje Tutorados & & 0 & 0 \\
\hline 2. Maestro-psicomotricista & & 0 & 0 \\
\hline 2.1. Aportación de la evaluación formativa al proceso de aprendiz & & 1 & 1 \\
\hline 2.2. Coherencia entre la tutorización y la evaluación formativa & & 1 & 1 \\
\hline 2.3. Instrumento de evaluación utilizado & & 1 & 5 \\
\hline 2.4. Participación del estudiante & & 1 & 2 \\
\hline 2.5. Tiempo destinado a la evaluación & & 1 & 1 \\
\hline 3. Estudiantes & & 0 & 0 \\
\hline 3.1. Aportación de la evaluación formativa al proceso de aprendiz & & 1 & 7 \\
\hline 3.2. Coherencia entre la tutorización y la evaluación formativa & & 1 & 3 \\
\hline 3.3. Instrumento de evaluación utilizado & & 1 & 2 \\
\hline 3.4. Participación del estudiante & & 0 & 0 \\
\hline 3.5. Tiempo destinado a la evaluación & & 1 & 2 \\
\hline 1. Profesor universidad & & 0 & 0 \\
\hline 1.1. Aportación de la evaluación formativa al proceso de aprendiz & & 1 & 2 \\
\hline 1.2. Coherencia entre la tutorización y la evaluación formativa & & 1 & 1 \\
\hline 1.3. Instrumento de evaluación utilizado & & 1 & 6 \\
\hline 1.4. Participación del estudiante & & 1 & 2 \\
\hline 1.5. Tiempo destinado a la evaluación & & 1 & 5 \\
\hline
\end{tabular}

Figura 1. Número de referencias para cada categoría e indicador (imagen extraída del programa Nudist N-Vivo 11)

Fuente: Elaboración propia.

A continuación, se muestra el análisis cualitativo de las categorías como resultados de la transcripción de los grupos de discusión. 


\subsection{Categoría: "Aportación de la evaluación formativa al proceso de aprendizaje"}

En esta categoría toma una especial relevancia los estudiantes ya que son los que más es dónde se encuentran más referencias relacionadas con la aportación de la evaluación formativa en su propio proceso de aprendizaje.

El estudiantado percibe la evaluación realizada en el PAT como una forma de aprendizaje que permite la identificación de los errores llevados a cabo durante todo el proceso y, posteriormente, posibilita que el estudiantado pueda rectificarlos y mejorarlos. Este seguimiento procedimental es valorado positivamente en aportaciones como:

Que no sea solo una evaluación cuantitativa, sino que también sea cualitativa, que podamos saber qué podemos mejorar, qué hemos fallado. (Estudiante 1)

También, los estudiantes valoran gratamente la coevaluación realizada a lo largo de la asignatura con sus compañeros al evaluar conjuntamente la sesión de psicomotricidad realizada en la universidad. Este hecho es debido a que se considera que el feedback formativo producido entre iguales se ha caracterizado por opiniones constructivas, favoreciendo la mejora del proceso de aprendizaje y aportando diferentes puntos de vista:

Es positivo las ideas que te dan porque están en el mismo nivel que tú y piensan que esta actividad puede ir mejor así... ¿Es un punto a favor no? (Estudiante 10)

En este aspecto específico sobre el valor de la coevaluación coinciden con el profesorado de la universidad, quienes cuando se les pregunta sobre los puntos clave del sistema de evaluación del PAT indican que:

Coevaluación entre estudiantes y la heteroevaluación, no solo entre profesora uni y estudiante, sino que también con el maestro-psicomotricista. (Profesora universidad 1)

Otro de los aspectos clave que destaca sobre la evaluación de este proyecto es el feedback formativo. El profesorado universitario pone en relieve el papel de éste en el PAT a partir de la valoración que les ha hecho llegar el propio estudiantado. Exponen que éstos les han transmitido que el feedback recibido tanto por el profesorado universitario como por los maestros-psicomotricistas es positivo ya que les permite situarse en el momento en el que se encuentran:

Valoran mucho que este feedback y este poder compartir después de la sesión sobre lo que se le ha dicho, de cómo había ido o no (en la medida en que hemos podido, eh). Pero yo creo que eso ellos lo valoran muchísimo y lo agradecen. $\Upsilon$ creo que no lo encuentran en otros lugares u otras asignaturas. $\Upsilon$ pienso que lo deberíamos retomar. (Profesora universidad 1)

Por último, los maestro-psicomotricistas destacan que la evaluación llevada a cabo en el PAT permite al estudiantado autoreflexionar y, a su vez, lo considera uno de los puntos clave del sistema de evaluación.

\subsection{Categoría: "Coherencia entre la tutorización y la evaluación formativa".}

En relación con el agente que realiza el acompañamiento de los estudiantes en los diferentes momentos del PAT y la evaluación que éste realiza, los estudiantes de la UAB reflexionan sobre la importancia de que su evaluación sea llevada a cabo por el docente que le ha acompañado. Cabe puntualizar que en esta universidad el grupo-clase se dividió en tres subgrupos y la profesora de la asignatura se encargó de la evaluación de la exposición final del PAT de solamente uno de los grupos y de los otros dos se encargaron maestros-psicomotricistas. Éstos tenían en su subgrupo a estudiantes que habían acompañado en las sesiones de psicomotricidad de su escuela y a otros que estuvieron en 
otros centros. La profesora de la asignatura justificó este hecho por falta de tiempo en el cronograma de la asignatura:

Como no había suficiente tiempo, que decimos que está todo muy compactado en la asignatura... (...) Pero al dividirlo, ella (señala a maestro-psicomotricista 1) se encargó de uno, ella (maestro-psicomotricista 2) de un otro y yo de otro. (Profesora universidad 1)

Los dos maestros-psicomotricistas que se vieron involucrados en esta situación comentan al respecto que se sintieron incómodos debido a la poca información que tenían al respecto por parte de la profesora de la asignatura:

Me sentí perdida, pero muy perdida. Porque primero, que no sabía que me tocaría. To fui allí como que iba a ver qué hacia la profesora 1... (...). Y entonces claro, llegas allí y te dicen 'te toca este grupo..." y claro, no sabias qué tenías que hacer. Entonces, te encuentras que eres tú la que tenías que llevar a ese grupo, ipero no sabía qué hacer! (Maestro-psicomotricista 1)

Frente a esta situación, el estudiantado muestra su desagrado y piden que exista coherencia entre la persona que les realiza el acompañamiento y la que finalmente les evalúa su PAT. Destacan que no les parece correcto que lo haga una persona que no ha podido conocer su proceso:

Me resultó poco agradable encontrarme a una persona alli que no había participado en el proceso (...). Encontrarte una persona allí que te está evaluando por ejemplo que no sabía de qué iba. (...). Nos tuvimos que dividir y se notaba que faltaba una persona que había participado en aquello más directamente. (Estudiante 6)

Los estudiantes agradecen una atención y trato de alguien que conoce y sigue su trabajo y que lo haga de forma personalizada. De esta forma el profesor puede realizar un seguimiento más individualizado de su proceso de aprendizaje:

Ya que hemos estado hablando del feedback, pienso que somos muchas alumnas y claro... el hecho de tener este momento contigo, el profesor también te ayuda a que te conozcas mejor (...) entonces claro, de ser tantas y a tener este momento más personal, yo pienso que es muy positivo. (Estudiante 8)

Destacar que frente a esta situación surgió un consenso entre las tres partes donde consideraban que lo ideal sería que tanto el profesorado de la asignatura (que acompaña al estudiantado en el diseño de la sesión de psicomotricidad con los adultos) como los maestros-psicomotricistas (que acompañan al estudiantado en la implementación de la sesión de psicomotricidad con los niños y niñas) evaluaran también, además de los diferentes procesos del PAT, la exposición final de éste pero en este caso de manera conjunta:

Para captar el sentido, yo creo que está clarísimo que deberíamos de haber estado el maestropsicomotricista y yo. Esto es lo correcto y esta era la intención, pero llega un momento que el tiempo... dices... jes que no tengo más! (Profesora universidad 1)

Pero que estuviera la profesora de referencia para hacerlo y no solo la maestra de la escuela. (Estudiante 3)

En el caso de la UdG se produjo una situación completamente diferente ya que tanto el profesorado de la asignatura como los maestros-psicomotricistas se reunieron previamente a la exposición final del PAT para comentar impresiones y realizar una evaluación conjunta que, después, compartieron también con los estudiantes. Profesorado y maestros-psicomotricistas valoraron positivamente esta coordinación ya que creen que aportó calidad a la evaluación: 
Nosotros sí que nos habiamos visto un día fuera, nos habiamos encontrado antes y como sería esta evaluación. Fue una evaluación muy genérica de sus grupos (señala maestropsicomotricista 5) y con su grupo (señala a maestro-psicomotricista 7). $Y$ sí que pactamos un poco como sería. (Profesora asignatura 2)

\subsection{Categoría: "Instrumentos de evaluación utilizados"}

Por lo que se refiere a los instrumentos utilizados para llevar a cabo la evaluación, durante los grupos de discusión también se debatió sobre la utilidad de éstos y se destaca que esta categoría es el más referenciado entre los tres participantes.

En cuanto al instrumento utilizado por el profesorado de la asignatura para evaluar la práctica docente individual de los estudiantes cuando dirigen la sesión de psicomotricidad con sus compañeros de la universidad, las profesoras no acaban de sentirse a gusto con él y proponen revisarlo para su mejora:

To creo que el instrumento que nosotras (refiriéndose a la profesora universidad 2 y a ella misma) hacíamos servir después de la sesión que era más individual y así... Sí que había como unos apartados que a mí me costaron un poco (profesor universidad 2 asiente) porque prácticamente es lo mismo todo. To creo que este lo deberíamos de revisar un poco más porque aún no está suficientemente bien. (Profesor universidad 1)

En cuanto al instrumento utilizado por los maestros-psicomotricistas para evaluar la sesión de psicomotricidad que los estudiantes diseñan y desempeñan en sus escuelas, cabe destacar que las sensaciones son diferentes a la del profesorado de la universidad. Éstos se muestran satisfechos con su instrumento y lo consideran muy útil y práctico ya que incluso les ha permitido revisar su propia práctica:

\footnotetext{
A mi el instrumento, el que nosotros teníamos que rellenar para poder evaluar, ¿sí? A mi éste sí que me fue muy bien porque te facilitaba que es aquello que tenías que mirar. Era muy fácil, ¿no? Además, como ponía en negrita las preguntas... (...) ¡Es que estaba todo! To creo que estaba todo, todo lo que tenías que mirar en una sesión, estaba allí. Por eso digo que a mí también me ha servido como autoevaluación. Yo también me he autoevaluado. (Maestropsicomotricista 1)
}

Aunque la opinión general respecto a este instrumento era muy satisfactoria, los maestrospsicomotricistas también estaban de acuerdo en que las posibles mejoras que se requerían para una próxima intervención. En concreto, destacaron que la evaluación cuantitativa que requería el instrumento les resultó difícil y les causó ciertas incongruencias:

Había la parte numérica que era como difícil, ¿no? ¡O difícil poner todo el grupo un mismo número! (...) Porque decía es injusto que diga pongo un dos porque aquí ha pasado una cosa $q u e . .$. (Maestro-psicomotricista 1)

Por su parte, el profesorado de la asignatura de la UdG expone que previamente al uso de este instrumento se reunió con los maestros-psicomotricistas para compartir qué significaba la calificación numérica de cada ítem para llegar a un consenso ya que les parecía que la evaluación resultaría más justa. Además, para los maestros-psicomotricistas uno de los puntos débiles de la evaluación del PAT precisamente era las diferentes interpretaciones que podría darse a los ítems cuantitativos de la evaluación y este colectivo propuso poder elaborar rúbrica para poder solventar esta situación:

Lo que nosotros sí que hicimos es encontrarnos un día y hablarlo. $\Upsilon$ dijimos qué es un 2, qué es un 3 y qué es un 4. (...). $\Upsilon$ lo consensuamos. Porque... ¿̇qué es un 2 ? $\Upsilon$ lo hicimos así. $\Upsilon$ entonces de cada pregunta, nos encontramos los cuatro para tener una evaluación más justa, ¿no?”(Profesora asignatura 2) 
Referente a este instrumento, destaca el contraste de opinión entre los estudiantes y los maestros-psicomotricistas. Mientras éstos últimos se encontraban satisfechos con su utilidad, el estudiantado expone que desconocía la existencia de éste y que no lo vieron en ningún momento.

El siguiente instrumento analizado es el utilizado por los estudiantes para autoevaluar el desarrollo de las competencias profesionales psicomotrices desarrolladas a través del PAT. El estudiantado expone que es un instrumento muy útil, que les permite conocerse mejor a partir de la propia conciencia y que les resultaba muy ventajoso la parte cualitativa de éste, donde podían reflexionar sobre su propia evolución de manera reflexiva y autocrítica:

Es muy positivo que se tenga que explicar el porqué de cada cambio a nivel cualitativo (...). Si antes tenías un 'nada' y ahora un 'mucho', tiene que haber un porqué, ¿̨este cambio porqué se hace? Esta reflexión, esta autocrítica, para ver qué he aprendido o no he aprendido, y tu darte cuenta. (Estudiante 6)

Por lo que se refiere a la cantidad de veces que los estudiantes debieron contestar la escala de autoevaluación, cabe decir que éstos tuvieron que hacerlo en tres momentos del desarrollo del PAT (inicio asignatura, después de la sesión práctica con los compañeros de universidad y después de la sesión práctica con los niños y niñas en la escuela). Tanto los estudiantes como el profesorado universitario consideran que contestándola al inicio y al final de la asignatura es suficiente para poder evaluar la adquisición de competencias profesionales psicomotrices ya que, contestándola a la mitad de la asignatura, los cambios producidos no eran muy significativos:

Habíamos pensado también que se lo hacemos rellenar en tres momentos y los importantes son el primero y el último, quizá el del medio no hace falta hacerlo, ¿no?. (Profesor universidad 1)

Por último, referente a la hipótesis de la sesión de psicomotricidad que el alumnado tenía que realizar, las tres categorías coinciden que es una herramienta muy útil para el proceso de aprendizaje del estudiante dentro del PAT, porque les permite reflexionar previamente sobre aquello que puede suceder en la sesión. Además, el maestro-psicomotricista considera que gracias a este instrumento le es más fácil iniciar un feedback, una vez finalizada la sesión en su centro educativo:

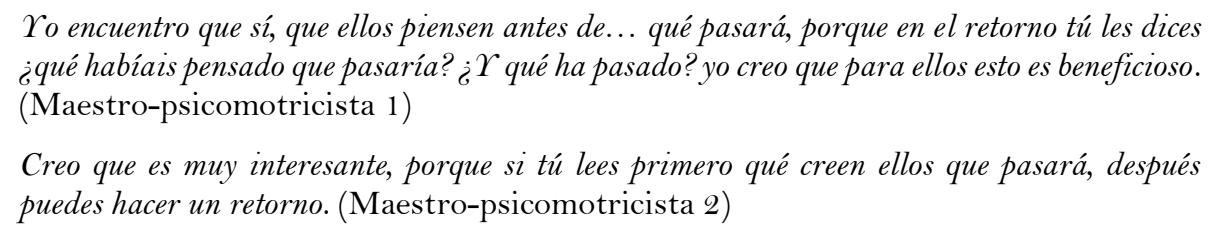

No obstante, en la práctica hubo algunas discrepancias ya que las hipótesis se presentaron al profesorado de la universidad (previamente a la sesión impartida con sus compañeros de la asignatura), pero algún grupo de estudiantes no la presentó a los maestrospsicomotricistas:

Mis grupos no lo tenían claro. Decían que no le habias dicho (refiriéndose al profesor universidad 1) que tenían que entregar la hipótesis. (Maestro-psicomotricista 3)

\subsection{Categoría: "Participación del estudiante"}

El profesorado de la universidad coincide en el papel relevante que tiene la participación del estudiante para su propia evaluación formativa. Es por ello que en la sesión de 
psicomotricidad impartida en la universidad el profesorado destaca la importancia de hacer un feedback entre todo el grupo-clase (co-evaluación) y posteriormente entre la profesora y el grupo que ha expuesto de forma más concreta (heteroevaluación):

Una vez que se había recogido todo, con el instrumento que teníamos de evaluación de la sesión, yo los juntaba a todos y les decía lo que había visto de la sesión. (Profesor universidad 1)

En la UAB se llevó a cabo este doble feedback, no obstante, desde la UdG, por falta de tiempo, no pudieron realizarlo con cada grupo que exponía, siendo un factor a mejorar para poder destinar un tiempo de evaluación con los distintos grupos sobre cómo ha ido la sesión y en general sobre cómo ha ido todo el proceso del PAT:

\title{
A mí me hubiera gustado una parte que no he hecho por falta de tiempo. Es antes con ellos, hacer una evaluación por grupos. (Profesor universidad 2)
}

Referente a la participación del estudiante en la evaluación de la sesión en la escuela, el maestro-psicomotricista valora de forma positiva que en el feedback se le pregunte previamente al estudiantado cómo se han visto y cuál sería la valoración que se harían, una vez finalizada la sesión de psicomotricidad con los niños y niñas. Esta participación y reflexión del estudiante puede servirle al maestro-psicomotricista para conocer un poco más el papel de cada uno de los participantes en el grupo y en qué medida han colaborado en la sesión.

\begin{abstract}
A la hora de hacer el retorno, las valoraciones las realice con ellos. To les preguntaba ¿vosotros, que creéis o qué nota creéis que tenéis? Además, a mí me pasaba que los grupos aparte de ser numerosos y tener en mi caso poco espacio en la sala, me pasaba que en el mismo grupo unos intervenían y otros no. $Y$ claro, ¿̇esto en la nota?, ¿qué nota les pongo? Porque había alguno que no lo sabía (...) yo creo que también agradecen el feedback del momento y la nota. (Maestro-psicomotricista 3)
\end{abstract}

\subsection{Categoría: "Tiempo destinado a la evaluación"}

En la siguiente categoría se analizan diferentes aspectos del PAT que deben de mejorarse y que están relacionados con el tiempo.

En relación al tiempo disponible para la evaluación final del PAT, tanto el profesorado como el maestro-psicomotricista coinciden que les faltó tiempo para hacer un feedback con cada grupo que exponía la sesión de psicomotricidad en la universidad.

Referente a la escala de autoevaluación, el estudiantado coincide que era demasiado extensa y necesitaban mucho tiempo para contestarla, aunque valoran positivamente su contenido. El profesorado también estaría de acuerdo con este cambio en el instrumento.

Mis estudiantes me lo han dicho (...) que tienen mucho trabajo y aunque digamos que es un momento, jes trabajo! $\Upsilon$ yo también creo que es un poco largo. Creo que en un futuro nos lo tenemos que volver a mirar. (Profesor universidad 2)

Por otro lado, el estudiantado también valora que se les dejó poco tiempo para llevar a cabo esta escala de autoevaluación, y que el tiempo transcurrido entre los tres momentos de autoevaluación a lo largo del PAT, fue escaso, no dejando que hubiese un proceso de aprendizaje fuertemente diferenciado en el estudiante.

Los docentes también reflexionan sobre el contenido del tiempo en el PAT y sus puntos débiles para mejorar en un futuro, que serían: Falta de tiempo para realizar el feedback, poco tiempo para recibir muchos grupos en una misma escuela y poco tiempo destinado al feedback. 


\section{Discusión y Conclusiones}

Retomando el objetivo principal de la presente investigación, que es analizar las percepciones de profesorado, maestros-psicomotricistas y estudiantes sobre la evaluación formativa en PAT para facilitar la adquisición de competencias profesionales psicomotrices en la formación inicial de maestros, se puede concluir que, a partir de los datos obtenidos, se ha logrado describir dichas percepciones pretendiendo que estas aporten significatividad para la construcción del conocimiento en el campo educativo, más concretamente en la educación psicomotriz.

Un aspecto relevante de este estudio se encuentra en la utilización de una metodología mixta, enmarcada dentro de un paradigma interpretativo, proporcionando una visión más amplia del estudio. También cabe destacar la presentación de unos resultados a través de la triangulación entre los resultados cualitativos y cuantitativos y entre los tres informantes (estudiantes, profesorado universitario y maestros-psicomotricstas de escuelas).

En los estudiantes, los datos cuantitativos muestran que están bastante satisfechos de forma global, (3,14 sobre 4) en relación a la evaluación del PAT, y consideran que la evaluación realizada también favorece bastante la adquisición de competencias profesionales (3,41 sobre 4). Estos resultados coinciden con los del estudio de MartínezMínguez (2015) en el que el 94\% del alumnado consideraba entre bastante y mucho que existía relación entre PAT y competencias profesionales. Además, en el mismo estudio se apuntaba una posible relación de este dato con la calificación final en la asignatura (el 80,71\% habían obtenido notable, sobresaliente o matrícula de honor).

Respecto a si la evaluación planteada favorece la adquisición de competencias profesionales, los estudiantes de la UAB puntúan ligeramente por encima de los de la UdG ( $\mathrm{M}=3,52$ y $\mathrm{M}=3,23$, respectivamente) mostrándose como una diferencia estadísticamente significativa. Esto podría deberse por un lado a que son estudiantes de cuarto curso y no de segundo, lo que les da un bagaje general más amplio estando ya al final de su formación. Y por otro lado a que ya han cursado créditos de asignaturas de Practicum, con el acercamiento al mundo profesional que aportan a modo competencial.

A nivel cualitativo, el alumnado expone que el PAT les permite conocer aspectos a mantener y mejorar en futuras intervenciones psicomotrices. A revisar apuntan algunos factores respecto a la coherencia entre el acompañamiento del PAT y su evaluación. Esto puede deberse a que intervienen diferentes agentes en la evaluación lo que obliga a un gran conocimiento y coordinación. Por un lado, entre docentes (profesorado y maestros) y más teniendo en cuenta que era la primera vez que trabajaban y evaluaban juntos a los mismos estudiantes. Y por otro lado a nivel de coevaluación entre estudiantes, al no ser una práctica habitual durante su formación inicial. Panadero, Fraile, Fernández, CastillaEstévez y Ruiz (2019), en un estudio donde exploraban la evaluación en la Educación Superior española actual, apuntaban como conclusiones que la evaluación entre iguales y la autoevaluación son bastante raros, y que las prácticas evaluativas casi no cambian entre los cuatro cursos de formación inicial.

Los docentes también destacan su satisfacción global sobre la evaluación del PAT porque consideran como un factor positivo el uso de diferentes tipologías de evaluación como la coevaluación entre estudiantes o docente-estudiante. A su vez exponen que debería de 
replantearse en un futuro la falta de tiempo para realizar el feedback con los estudiantes y la dificultad que comporta la parte cuantitativa de los instrumentos de evaluación. De acuerdo con Colomer, Serra, Cañabate y Serra (2018), vemos la necesidad de incorporar la reflexión crítica por parte del alumnado en el planteamiento de futuras investigaciones. Para ello será necesario también tener en cuenta una formación por parte del alumnado y docentes, para que sea más reflexivos y críticos.

Los resultados obtenidos refuerzan los trabajos realizados por otros autores con estudios similares. Concretamente en relación a la satisfacción del alumnado, han aparecido elementos beneficiosos de esta modalidad evaluativa, que han aparecido también señalados en otros estudios recientes, (Galván y Farías, 2018; Gómez y Quesada 2017; Valvanuz y Salcines, 2018). Pero debemos tener en cuenta que para que las prácticas innovadoras tengan unos efectos deseables es necesario que sean sostenibles y continuas. Este es un aspecto que los autores de este estudio toman el compromiso.

Los estudiantes apuntan a que la mayor potencialidad del aprendizaje proviene de la valía de la retroalimentación que se ofrece y la posibilidad de ser más conscientes, reflexionar y aprender de los errores cometidos, aspecto que reafirma los trabajos presentados por Sant Martín (2012). Los resultados de este estudio coinciden con los realizados por MedinaRivilla, Domínguez-Garrido y Sánchez-Romero, (2013) y Rodríguez, Ibarra y Gómez (2011), que enfatizan y muestran también la necesidad de utilizar en el proceso de aprendizaje, procedimientos de autoevaluación y coevaluación entre profesorado y alumnado o recíprocamente entre alumnado, con objeto de poder alcanzar un aprendizaje más profundo a lo largo del proceso.

La coevaluación realizada a lo largo de la asignatura con sus compañeros/as al evaluar conjuntamente la sesión de psicomotricidad realizada en la universidad, ha sido valorado muy satisfactoriamente por considerarlo como un feedback formativo entre iguales que ha favorecido su proceso de aprendizaje, así como la capacidad de reflexión y autocrítica.

El alumnado valora muy positivamente la continuidad en el trabajo, la distribución de las tareas a lo largo del proceso y el seguimiento realizado por el profesor de la universidad ya que le permite establecer un contacto continuado e ir incrementando así su aprendizaje. Resultados similares pueden encontrarse también en otros trabajos (Fraile, López-Pastor, Castejón, y Romero, 2013; Hortigüela-Alcalá, Pérez-Pueyo y López-Pastor, 2015).

A lo largo de todo el proceso el alumnado se ha ido implicando más en la evaluación a medida que ha ido comprobando evidencias de su aprendizaje y ha podido tomar juicios de valor sobre su trabajo. Esto coincide con el estudio de Martínez-Mínguez (2016) en el que al inicio el estudiante piensa que no tiene ni idea de lo que implica la psicomotricidad. Pero al ir avanzando en formación teórica e ir poniendo en práctica las sesiones diseñadas, surge el cambio y se dan cuenta de lo que se ha aprendido.

Por lo que se refiere a los instrumentos utilizados para llevar a cabo la evaluación, la opinión general de todos los participantes ha sido satisfactoria, coincidiendo con Vallés, Martínez-Mínguez y Romero (2018) en que el profesorado universitario español aplica una amplia gama de herramientas de evaluación que permiten potenciar diferentes competencias del alumnado. También los docentes consideran que es aconsejable hacer una revisión para posibles mejoras. Sobre todo, en relación a facilitar la transmisión de los resultados. Algunos de los maestros-psicomotricistas se sentían más cómodos haciendo 
aportaciones cualitativas y uno de los puntos débiles de la evaluación del PAT precisamente era las diferentes interpretaciones que podría darse a los ítems cuantitativos de la evaluación. Para posteriores estudios se propone: rehacer el instrumento de evaluación de la práctica docente individual dotándolo de más concreción y una clara diferenciación entre aspectos a evaluar; consensuar previamente los criterios de calificación o crear rúbricas para eliminar sesgos de interpretaciones en el instrumento utilizado por los maestros-psicomotricistas para evaluar la sesión realizada en su escuela; y utilizar la escala de autoevaluación de competencias únicamente al inicio y final de la asignatura, eliminando la del momento central pues no se perciben cambios suficientemente diferenciadores.

En este estudio debemos hacer mención también de la importancia del rol del docente. Este ha sido fundamental para guiar el proceso y en la aplicación de feedbacks eficientes (Lin y Lai 2013), garantizando una mayor retención y aplicación del aprendizaje.

La finalidad de este estudio es que pueda ser de interés para el profesorado universitario que desee desarrollar sistemas de evaluación formativa en PAT en sus asignaturas con objeto de mejorar el proceso de aprendizaje. También pensamos que puede ser de interés para grupos de investigación educativa e innovación docente que estén trabajando esta temática.

Un aspecto relevante en esta investigación ha sido el poder llevar a cabo un proceso de reflexión conjunta a tres bandas (alumnos, maestros- psicomotricistas y profesores). El trabajo en equipo y la reflexión compartida han hecho más livianas las inseguridades que genera hacer algo diferente e innovador

El trabajo aquí presentado pretende ser un intento de avanzar en la construcción de una investigación educativa comprometida socialmente y profesionalmente, orientada a la calidad del aprendizaje.

Por último, quisiéramos resaltar algunas propuestas de mejora del presente trabajo y líneas futuras de investigación.

- Que el rol/papel/función de los maestros-psicomotricistas no solo sea observar la sesión que llevan a cabo los estudiantes en sus escuelas y luego les realicen un feedback después de ésta, sino que también puedan acompañarlos (junto con el profesorado de la asignatura) en el diseño de la sesión de psicomotricidad desde el conocimiento del propio contexto en concreto.

- Proponer en futuras intervenciones que los estudiantes puedan ir previamente a la escuela para conocer al psicomotricista en persona, las instalaciones y el material del cual se dispone. Y, complementariamente, quien quisiera también podría observar una sesión de psicomotricidad con el grupo de niños y niñas donde realizaran su intervención para poder tener un primer contacto y conocimiento de éstos.

- Invitar a los maestros-psicomotricistas a asistir el día que se presenta el PAT en la asignatura para presentarse personalmente, explicar su manera de trabajar, y algunas características más destacables de su escuela (instalaciones, material, características generales de los niños y niñas, etc.). 
- Elaborar un instrumento que sirva para poder realizar el diseño de la sesión con los niños y niñas por parte de los estudiantes y que facilite la implicación del maestro-psicomotricista en el acompañamiento y realización de ésta.

- Que la evaluación general del PAT se realice entre el profesorado de la universidad y los maestros-psicomotricistas. Por lo tanto, que los dos estén presentes el día de la exposición final del PAT ya que los dos han formado parte del proceso de aprendizaje de los estudiantes.

Para finalizar, debemos resaltar la importancia que tiene la actitud del docente en la puesta en práctica de experiencias. Si se desea tener posibilidades de éxito será necesario que exista un diálogo verdadero entre los protagonistas, que se establezca un marco de confianza y de respeto. Solo así se podrá fomentar una evaluación formativa, critica, inclusiva y justa, que permita un aprendizaje competencial.

\section{Agradecimientos}

Este estudio se ha llevado a cabo dentro de dos proyectos de investigación competitivos:

- Mejora e innovación en la formación psicomotriz inicial de maestros. Análisis de mecanismos de vinculación de aprendizajes teóricos con práctica real en las escuelas haciendo uso de metodologías activas y evaluación formativa. Convocatoria de Ayudas de investigación en mejora e innovación en la formación inicial de maestros para las titulaciones impartidas en las universidades participantes en el Programa de Mejora e Innovación en la Formación de Maestros (ARMIF 2014), de la Agencia de Gestión de ayudas Universitarias y de Investigación (AGAUR). Convocatoria ECO / 2788/2013. Referencia: 2014-ARMIF-00031. Modalidad: 2.

- Las competencias docentes en la formación inicial del profesorado de educación física. Del Programa Estatal de Investigación, Desarrollo e Innovación Orientada a los Retos de la Sociedad, en el marco del Plan Estatal de Investigación Científica y Técnica y de Innovación 2013-2016. Referencia: EDU 2013-42024-R.

\section{Referencias}

Albert, M. J. (2007). La investigación educativa. Claves teóricas. Madrid: McGraw-Hill.

Álvarez, J. M. (2003). La evaluación a examen. Madrid: Miño y Dávila.

Álvarez, I. (2008). Evaluación del aprendizaje: una Mirada retrospectiva y prospectiva desde la divulgación científica. Revista Electrónica de Investigación Psicoeducativa, 14(1), 235-272.

Álvarez Rojo, V., García Jiménez, E., Gil Flores, J. y Romero Rodríguez, S. (2004). La enseñanza universitaria: Planificación y desarrollo de la docencia. Almería: EOS.

Angulo, J. F. (2008). La voluntad de distracción: las competencias en la universidad. En J. Jimeno Sacristán (Ed.), Educar por competencias, ¿̨que hay de nuevo? (pp. 175-205). Madrid: Morata.

Barba, J. J., Martínez, S. y Torrego, L. (2012). El proyecto de aprendizaje tutorado cooperativo. Una experiencia en el grado de maestra de Educación Infantil. Revista de Docencia Universitaria. REDU, $1 O(1), 123-144$. 
Battaglia, M. P. (2008a). Convenience sampling. Encyclopedia of Survey Research Methods. Londres: SAGE Publications.

Battaglia, M. P. (2008b). Nonprobability sampling. Encyclopedia of Survey Research Methods. Londres: SAGE Publications.

Biggs, J. B. (2005). Calidad del aprendizaje universitario. Madrid: Narcea.

Bisquerra, R. (2014). Metodología de la Investigación Educativa. Madrid: La Muralla.

Bonsón, M. y Benito, A. (2005). Evaluación y Aprendizaje. En A. Benito y A. Cruz (Eds.), Nuevas claves para la docencia universitaria en el Espacio Europeo de Educación Superior (pp. 87-100). Madrid: Narcea.

Brockbank, A. y Mcgill, I. (2002). Aprendizaje reflexivo en la educación superior. Madrid: Morata.

Brown, S. y Glasner, A. (2000). Assessment matters in higher education: choosing and using diverse approaches. Buckingham: The Society for Research into Higher Education.

Bretones, A. (2008). Participación del alumnado de Educación Superior en su evaluación. Revista de Educación, 347, 181-202.

Bueno, D. (2017). Neurociencia para educadores. Barcelona: Octaedro.

Comunicado de Berlín. (2003). Educación Superior Europea. Recuperado de http://www.eees.es/pdf/Berlin_ES.pdf

Cano, E. (2012). Aprobar o aprender. Estrategias de evaluación en la sociedad red. Barcelona: Laboratori de Mitjans Interactius, Universitat de Barcelona.

Castejón Oliva, F. J., Santos Pastor, M. L. y Palacios Picos, P. (2015). Cuestionario sobre metodología y evaluación en formación inicial en educación física. Revista Internacional de Medicina y Ciencias de la Actividad Física y el Deporte, 15, 245-267.

Cañabate, D., Colomer, J. y Oliveras, J. (2018). Movement: A Language for Growing. Apunts. Educación Física y Deportes, 134, 146-155. https://doi.org/10.5672/apunts.20140983.es.(2018/4).134.11

Colomer, J., Serra, L., Cañabate, D. y Serra, T. (2018). Evaluating and assessment-centred reflective-based learning approaches. Sustainability, 10, 31-22. https://doi.org/10.3390/su 10093122

Creswell, J.W. (2009). Research desing: Qualitative, quantitative and mixed approaches. Thousand Oaks, CA: Sage.

Declaración de Bolonia. (1999). El Espacio Europeo de la Enseñanza Superior. Recuperado de http://www.eees.es/pdf/Bolonia_ES.pdf

Del Rincón, D., Arnal, J., Latorre, A. y Sans, A. (1995). Técnicas de investigación en ciencias sociales. Madrid: Dykinson.

Echeverría, B. (2002). Gestión de la competencia de acción profesional. Revista de Investigación Educativa, 20(1), 7-43.

Escudero, T. (2010). Sin tópicos ni malentendidos: fundamentos y pautas para una práctica evaluadora de calidad en la enseñanza universitaria. Zaragoza: ICE de la Universidad de Zaragoza.

Eurydice. (2002). Las competencias clave. Madrid: MECD Ministerio de Educación Ciencia y Cultura. 
Ferguson, P. (2011). Student perceptions of quality feedback in teacher education. Assessment $\mathbb{F}^{\circ}$ Evaluation in Higher Education, 36(1), 51-62. https://doi.org/10.1080/02602930903197883

Fraile, A., López-Pastor, V., Castejón, J. y Romero, R. (2013). La evaluación formativa en docencia universitaria y el rendimiento académico del alumnado. Aula Abierta, 41(2), 23-34.

Galván, J. O. y Farías, G. M. (2018). Características personales y práctica docente de profesores universitarios y su relación con la evaluación del desempeño. Revista Iberoamericana de Evaluación Educativa, 11(2), 9-33.

García-Sanz, M. P. (2014). La evaluación de competencias en Educación Superior mediante rúbricas: un acaso práctico. Revista Electrónica Interuniversitaria de Formación del Profesorado, 17(1), 87-106. https://doi.org/10.6018/reifop.17.1.198861

Gibbs, G. (2012). El análisis de datos cualitativos en investigación cualitativa. Madrid: Morata.

Gómez, M. A. y Quesada, V. (2017). Coevaluación o evaluación compartida en el contexto universitario: la percepción del alumnado de primer curso. Revista Iberoamericana de Evaluación Educativa, 1O(2), 9-30. https://doi.org/10.15366/riee2017.10.2.001

Guilarte, C., Marbán, C. y Miranda, J. M. (2008). Principios básicos para el diseño de guías docentes de asignaturas en el marco del EEES. Valladolid: Universidad de Valladolid.

Hernández, R., Fernández, C. y Baptista, P. (2010). Metodología de la investigación. Ciudad de México: McGraw-Hill.

Hortigüela-Alcalá, D., Pérez-Pueyo, A. y López-Pastor, V. (2015). Implicación y regulación del trabajo del alumnado en los sistemas de evaluación formativa en educación superior. RELIEVE, 21(1) https://doi.org/10.7203/relieve.21.1.5171

Ibarra, M. S., Rodríguez, G. y Gómez, M.A. (2012). La evaluación entre iguales: beneficios y estrategias para su práctica en la universidad. Revista de Educación, 359, 206-231.

Inda, S., Álvarez, S., y Álvarez, R. (2008). Métodos de evaluación en la enseñanza superior. Revista de Investigación Educativa, 26(2), 539-552.

Jarman, K. H. (2013). The art of data analysis: How to answer almost any question using basic statistics. Hoboken, NJ: Wiley.

Lin, J. y Lai, Y. (2013). Harnessing. Collaborative Annotations on Online Formative Assessments. Educational Technology \& Society, 16(1), 263-274.

López-Pastor, V. (2009). Evaluación formativa y compartida en Educación Superior. Madrid: Narcea.

López Ruiz, J. I. (2011). Un giro copernicano en la enseñanza universitaria: formación por competencias. Revista de Educación, 356, 279-301.

López, M. A. y Vicente, F. (2015). Proyecto de aprendizaje tutorado en la formación científica inicial de las estudiantes del Grado Educación Infantil. En N. González, I. Salcines y E. García (Eds.), Tendencias emergentes en evaluación formativa y compartida en docencia. El papel de las nuevas tecnologías. Actas IX Congreso Internacional de Evaluación Formativa y Compartida en Docencia Infantil, Primaria, Secundaria y Universitaria (pp. 959-978). Santander: Universidad Cantabria.

López-Pastor, V. M. (2009). Evaluación formativa y compartida en educación superior: propuestas, técnicas, instrumentos y experiencias. Madrid: Narcea. 
Martínez-Mínguez, L. (2016). Proyectos de Aprendizaje Tutorados y autoevaluación de competencias profesionales en la formación inicial del profesorado. Retos. Nuevas Tendencias en Educación Física, Deportes y Recreación, 29, 242-250.

Martínez-Mínguez, L. (2015). Proyecto docente psicomotriz: «Buena práctica» en el Grado de Educación Infantil de la UAB. En N. González, I. Salcines y E. García (Eds.), Tendencias emergentes en evaluación formativa y compartida en docencia. El papel de las nuevas tecnologías. Actas IX Congreso Internacional de Evaluación Formativa y Compartida en Docencia Infantil, Primaria, Secundaria y Universitaria (pp.1093-1120). Santander: Universidad Cantabria.

Martínez-Mínguez, L. y Moya, L. (2017). La opinión de los estudiantes: la tutoría académica en la adquisición de competencias profesionales de educación física en los graduados en Educación Infantil. Didacticae, 2, 71-88.

Martínez-Mínguez, L., Rota, J. y Anton, M. (2017). Psicomotricitat, Escola i Currículum. Barcelona: Octaedro.

Martínez-Mínguez, L., Forcadell, X., Moya, L., Heras, G., Bru, E., Llecha, M., Sánchez, C., Pérez, M. y Anton, M. (2017). Psicomotricistas reflexionan sobre la educación por el movimiento. Entre Líneas. Revista especializada en Psicomotricidad, 39, 5-13.

Mas, O. (2011). El profesor universitario: sus competencias y formación. Profesorado. Revista de Curríuclum y Formación del Profesorado, 15(3), 195-211.

MEC. (2003). La integración del sistema universitario español en el Espacio Europeo de Enseñanza Superior. Recuperado de http://www.eees.es/pdf/Documento-Marco_10_Febrero.pdf.

Medina Rivilla, A., Domínguez Garrido, M.C. y Sánchez Romero, C. (2013). Evaluación de las competencias de los estudiantes: modelos y técnicas para la valoración. Revista de Investigación Educativa 31(1), 239-255.

Meyer, V. (2002). Project oriented learning (POL) as a communication tool of environmental sciences in the community of Sohanguve. A case study. Comunicación presentada en el International Conference on Sustainability of Water Resources, Universidad de Murdoch, Australia.

Miles, M. B. y Huberman, A. M. (1994). Qualitative Data Analysis: A Sourcebook of New Methods. Newbury Park, CA: Sage.

OCDE. (2002). DeSeCo (Definition and Selection of Competences). Recuperado de http://www.oecd.org/education/skills-beyond-school/41529556.pdf

Orden ECI/3960/2007, de 19 de diciembre, por la que se establece el curriculum y se regula la ordenación de la educación infantil, Boletín Oficial del Estado, 5, 1016-1036 (2007). Recuperado de https://www.boe.es/boe/dias/2008/01/05/pdfs/A01016-01036.pdf

Panadero, E., Fraile, J., Fernández, J., Castilla-Estévez, D. y Ruiz, M. A. (2019). Spanish university assessment practices: examination tradition with diversity by faculty. Assessment \& Evaluation in Higher Education, 44(3), 1-19.

https://doi.org/10.1080/02602938.2018.1512553

Perrenoud, P. (2004). Diez nuevas competencias para enseñar. Barcelona: Graó.

Rodríguez, G., Gil, J. y García, E. (1999). Metodología de la investigación cualitativa. Málaga: Aljibe.

Rodríguez, G., Ibarra, M. S. y Gómez, M. A. (2011). e-Autoevaluación en la universidad: Un reto para profesores y estudiantes. Revista de Educación, 356, 401-430.

Romero Martín, R., Fraile Aranda, A., López Pastor, V. M. y Castejón Oliva, F. J. (2014). The relationship between formative assessment systems, academic performance and teacher and 
student workloads in higher education. Infancia y Aprendizaje: Journal for the Study of Education and Development, 37(2), 310-341. https://doi.org/10.1080/02103702.2014.918818

Rust, C. (2007). Towards a scholarship of assessment. Assessment and Evaluation in Higher Education, 32(2), 229-237.

Sadler, D. R. (2010). Beyond feedback: Developing student capability in complex appraisal. Assessment and Evaluation in Higher Education, 35(5), 535-550. https://doi.org/10.1080/02602930903541015

Salinas, D. (2002). ¡Mañana examen!: la evaluación entre la teoría y la realidad. Barcelona: Graó.

San Martín, C. (2012). Atención a la diversidad en el contexto educativo chileno: concepciones del profesorado sobre evaluación y diseño de la propuesta curricular. REICE. Revista Iberoamericana sobre Calidad, Eficacia y Cambio en Educación, 1O(4), 165-183.

Sassano, M. (2010). El cuerpo como eje transversal en la escuela. En P. Bottini (Comp.), La psicomotricidad: práctica y conceptos (pp. 225-249). Buenos Aires: Miño y Dávila.

Taras, M. (2002). Using assessment for learning and learning from assessment. Assessment and Evaluation in Higher Education, 27(6), 501-510. https://doi.org/10.1080/0260293022000020273

Taras, M. (2010). Student self-assessment: processes and consequences. Teaching in Higher Education, 15(2), 199-209.

Torrance, H. (2007). Assessment as Learning? How the use of explicit learning objectives, assessment criteria and feedback in post-secondary education and training can come to dominate learning. Assessment in Education: Principles, Policy \& Practice, 14(3), 281-94.

Trenchi, N. (2014). TICs, Salud Mental y Crianza. Primer Encuentro sobre el uso saludable de las TICs $y$ redes sociales en la infancia $y$ adolescencia. Recuperado de https://www.youtube.com/watch?v=tL9g2oS13rM

Trevitt, C., Breman, E. y Stocks, C. (2012). Assessment and learning: Is it time to rethink student activities and academic roles? Revista de Investigación Educativa, 30(2), 253- 269.

Valvanuz, V. y Salcines, I. (2018). Estudio sobre la Implementación de la Evaluación Formativa y Compartida en un Ciclo Formativo de Grado Superior. Revista Iberoamericana de Evaluación Educativa, 11(2), 91-112. https://doi.org/10.15366/riee2018.11.2.005

Vallés, C., Martínez-Mínguez, L. y Romero, M. R. (2018). Instrumentos de evaluación: Uso y competencia del profesorado universitario en su aplicación. Estudios Pedagógicos, 44(2), 149169. https://doi.org/10.4067/So7 18-07052018000200149

Zabalza, M. A. (2002). Diseño curricular en la universidad. Competencias del docente universitario. Madrid: Narcea.

\section{Cv de las autoras}

\section{Lurdes Martínez-Mínguez}

Profesora Agregada. Departamento Didáctica Expresión Musical, Plástica y Corporal. Coordinadora de: Grado en Educación Infantil, Grupo de Investigación en Educación Psicomotriz (2017-SGR-139), especialidad de Arte, Cuerpo y Movimiento Máster Universitario de Investigación en Educación, Postgrado en Desarrollo Psicomotor de 0 a 
8 años. Miembro de la Red Nacional de Evaluación Formativa y Compartida. Sus líneas de investigación giran alrededor de: Evaluación formativa en la formación inicial de maestros; Desarrollo y evaluación de competencias profesionales psicomotrices; Encage de la psicomotricidad en los centros educativos; y Formación específica profesionalizadora e investigadora del psicomotricista. Es investigadora principal y participa en algunos proyectos de investigación e innovación docente. Es autora de diversos artículos indexados (JCR, SCOPUS, etc.) y de libros. ORCID ID: 0000-0003-1775-6288. Email: lurdes.martinez@uab.cat

\section{Laura Moya Prados}

Personal Investigador en Formación. Departamento Didáctica Expresión Musical, Plástica y Corporal. Coordinadora de Postgrado en Desarrollo Psicomotor de 0 a 8 años. Miembro del Grupo de Investigación en Educación Psicomotriz (2017-SGR-139). Miembro de la Red Nacional de Evaluación Formativa y Compartida. Sus líneas de investigación giran alrededor de: Evaluación formativa en la formación inicial de maestros; Desarrollo y evaluación de competencias profesionales psicomotrices; Encage de la psicomotricidad en los centros educativos; y Formación específica profesionalizadora e investigadora del psicomotricista. Participa en algunos proyectos de investigación e innovación docente. Y dispone de algunas publicaciones indexadas a nivel nacional. ORCID ID: 0000-0001-8324-4139. Email. Laura.Moya@uab.cat

\section{Carolina Nieva Boza}

Profesora asociada en el Departamento Didáctica Expresión Musical Plástica y Corporal de la UAB. Doctora en Actividad Física, Educación Física y Deporte. Profesora en el Postgrado en Desarrollo Psicomotor de 0 a 8 años y en el Máster Universitario de Investigación en Educación. Miembro del grupo de "Investigación en Educación Psicomotriz" (2017-SGR-139). Miembro de la "Red de Evaluación Formativa y Compartida en la Educación Superior" (REFYCES). Participa en el proyecto de "Autoevaluación de competencias de los estudiantes y desarrollo profesional de los maestros. Mejora de la práctica e innovación docente trabajando por proyectos de aprendizaje psicomotores co-tutorados universidad-escuela-(2017-ARMIF-00013). Ha publicado en las revistas de impacto Apuntes (2018) y Sport, Education and Society (2018). Ha participado en congresos internacionales como CIMIE, CIEF, CIAFC, en jornadas, workshops, etc. ORCID ID: 0000-0002-8593-1183. Email: carolina.nieva@uab.cat

\section{Dolors Cañabate Ortiz}

Profesora Agregada en el Área de Didáctica de la Expresión Corporal del Departamento de Didácticas Específicas de la Facultad de Educación y Psicologia de la UdG. Doctora por la Universitat de Girona. Coordinadora la Red de Innovación Docente en aprendizaje cooperativo(XIDAC). Investigadora principal del Grupo de investigación Consolidado GREPAI. Participa actualment en diversos proyectos de investigación e innovación docente. Ha desarrollado investigaciones principalment a lo largo de dos líneas: 1) Aprendizaje reflexivo y cooperativo. Metodologies activas y participatives. 2) movimiento y lenguajes: fomentar todas las ciencias que utilizan elementos del cuerpo como una 
estrategia, desde una perspectiva integral en el campo de la educación. Es autora de diversos articulos indexados (JCR; SCOPUS y otros), y de libros en el campo de la Educación. ORCID ID: 0000-0003-2526-9818. Email: dolors.canyabate@udg.edu 